\title{
Conceptual equivalence and health-related quality of life: an exploratory study in Japanese and Dutch cancer patients
}

\author{
W. Chr. Kleijn ${ }^{1,2}$, K. Ogoshi ${ }^{3}$, K. Yamaoka ${ }^{4}$, T. Shigehisa ${ }^{5}$, Y. Takeda ${ }^{6}$, C. L. Creutzberg ${ }^{7}$, \\ J. W. R. Nortier ${ }^{7}$ \& A. A. Kaptein ${ }^{1}$ \\ ${ }^{1}$ Leiden University Medical Center, Medical Psychology, P. O. Box 9555, 2300 RB, Leiden, The Netherlands, \\ (E-mail: w.c.kleijn@lumc.nl); ${ }^{2}$ Centrum' 45, Rijnzichtweg 35, 2342 AX, Oegstgeest, The Netherlands; \\ ${ }^{3}$ Department of Surgery, School of Medicine, Tokai University, Tokyo, Japan; ${ }^{4}$ Department of Technology \\ Assessment and Biostatistics, National Institute of Public Health, Saitama, Japan; ${ }^{5}$ Department of Psychology, \\ Tokyo Kasei Gakuin University, Tokyo, Japan; ${ }^{6}$ Department of Pulmonary Medicine, International Medical \\ Center of Japan, Tokyo, Japan; ${ }^{7}$ Department of Clinical Oncology, Leiden University Medical Center, Leiden, \\ The Netherlands
}

Accepted in revised form 1 February 2006

\begin{abstract}
Research into the equivalence of Western and Japanese conceptualizations of health-related quality of life (HR-QOL) is scarce. We used the Western (European Organization for Research and Treatment of Cancer, EORTC-QLQ-C30) and the Japanese (HRQoL-20) questionnaire in order to analyze the conceptual similarity of HR-QOL factors, and the associations between specific symptom items with overall HR-QOL in Japanese $(n=265)$ and Dutch $(n=174)$ patients with various types of cancer. Both populations completed both instruments. In both patient groups, the overall health scale of the EORTC-QLQ-C30 correlated highly $(r=0.59 ; p<0.001)$ with the HRQOL-20 composite average score, indicating substantial conceptual comparability. Relationships between all EORTC-QLQ-C30 symptom items with HR-QOL were examined by ranking their correlations with the two overall measures of HR-QOL. Comparable patterns in the Japanese and Dutch samples were observed. The results suggest a considerable conceptual equivalence of HR-QOL in Japanese and Dutch cancer patients, and indicate a satisfactory structural and cross-cultural equivalence for the EORTC-QLQ-C30 with regard to items measuring functioning and specific symptoms. Longitudinal studies are needed to examine the impact of specific symptoms on general quality of life.
\end{abstract}

Key words: Cancer, Health-Related Quality of Life, Conceptual equivalence, EORTC-QLQ-C30, HRQoL-20

\section{Introduction}

The growing number of internationally organized clinical trials and comparative studies in cancer patients corroborate the importance of crosscultural validation and standardization of healthrelated quality of life (HR-QOL) measurements
[1-3]. In order to examine the HR-QOL of cancer patients in a cross-culturally valid way, the European Organization for Research and Treatment of Cancer (EORTC) has developed various QOL questionnaires that have become standard HRQOL instruments. The EORTC-QLQ-C30 is a generic questionnaire in this set of measures [2]. 
This questionnaire has been used in more than 3000 studies worldwide. As a copyrighted instrument, it has been translated and validated into more than 50 languages, including Dutch and Japanese versions.

According to Naito et al. [1], researchers in English-speaking countries have pioneered the use and standardization of HR-QOL measurements. These authors state that clinicians in Japan reporting on QOL research have generally been interested in the use of methods that primarily measure the physical aspects of QOL. However, some Japanese researchers have evaluated the multidimensional QOL of cancer patients, employing either Japanese translations of Western measures, or HR-QOL instruments developed in Japan such as the HRQoL-20 [4-6].

Kobayashi et al. [4] translated the EORTCQLQ-C30 into Japanese and compared the scores of Japanese lung cancer patients with European patients. They stated that "both the Japanese language and culture are vastly different from those of English speaking countries", but confirmed the cross-cultural functional equivalence and concluded that this instrument can be used in international phase III studies. In his comments on the results of Kobayashi et al.'s study, Aaronson [7] draws attention to the particular importance of the relatively high correlations observed in the Japanese sample between scales assessing physical and psychological health domains, stating that "... one cannot rule out ... the cross-cultural explanation ... that the Japanese culture adheres less strongly to a Cartesian view of health than is the case in the West; that the illness experience of Japanese patients is of a more holistic nature." [7, p. 768]. To study cross-cultural equivalence, several dimensions were proposed by Hui and Triandis [8]: functional equivalence (adequacy of translation), scale equivalence (comparability of scales), operational equivalence (standardization of testing procedure), and metric equivalence (transferability of scoring results from one culture to another).

However Fayers et al. [9, 10] have pointed to two peculiarities that are characteristic of many health related QOL instruments, including the most widely used ones like the EORTC-QLQ-C30, which complicate the study of cross-cultural equivalence.
These instruments typically measure concepts using a combination of subscales and scale types. HRQOL subscales generally consist of a number of items measuring concepts such as emotional, physical, or social well-being, plus single-item scales measuring specific symptoms. Factor analytic methods are appropriate to examine structural characteristics of instruments that measure certain concepts. But in case of QOL instruments such as the EORTC-QLQ-C30, such methods are only useful for a subset of the items: only for the items defining certain constructs. Disease-specific QOL instruments, however, frequently contain items that assess symptoms or treatment side effects. These items may cause reductions in QOL-scores for those patients experiencing them, but the reverse relationship need not apply: a poor level of QOL does not imply that the patient suffers from that specific symptom. Hence, in this study we adopted the dataanalytic strategy proposed by Fayers et al. and use their method of distinguishing between different types of items. Following conventions from the field of structural equation modelling, these authors named the items belonging to subscales that primarily reflect the level of general QOL 'effect indicators'. These items are suitable to measure the level of HR-QOL. Items which typically assess specific disease or treatment symptoms (such as 'pain' or 'vomiting') are called a-priori, 'causal indicators', because the occurrence of these symptoms could cause a change in the level of general QOL.

Apart from this distinction between 'effect indicators' and 'causal indicators', we make a distinction between 'overall' QOL and 'domain specific' QOL in this study. Both QOL instruments used in this study measure domain specific QOL by using sets of conceptually related items measuring cognitive, emotional or social functioning. In addition, overall QOL is measured by one single item in the EORTC-QLQ-C30, while overall QOL is measured by the HRQoL-20 by calculating a mean score based on all 20 items. Our primary objective was to use the distinction in types of indicators and the different measures of HR-QOL in analyses of conceptual equivalence, a strategy which has not been used in previous cross-cultural studies.

As mentioned before few studies have focused on the issue of equivalence of conceptualizations 
of HR-QOL between Western and Japanese cancer patients. Japanese HR-QOL instruments have not yet been tested in Western populations of cancer patients. So their potential cultural bias or equivalence in measuring QOL is not known. However, the use of instruments from both cultures in a cross-cultural investigation can be highly informative. Therefore, in our study both Western and Japanese QOL instruments were employed to examine HR-QOL in both populations.

To summarize, this study had three aims: (1) to compare the relations of the specific symptom items of the EORTC-QLQ-C30 to measures of overall HR-QOL in Japanese and Dutch patients with the aim of analyzing conceptual equivalence; (2) to examine associations of quality of life with background variables such as country of origin, gender, age and illness stage; and (3) to describe and compare quality of life in Japanese and Dutch groups of cancer patients, using two different QOL scales developed in Japan and Europe.

\section{Methods \\ Procedure}

The data for an exploratory analysis on HR-QOL were selected from two ongoing Japanese and Dutch studies, both of which included patients with cancer [11, 12]. Because these studies used translated versions of the same HR-QOL instruments, and because both the HRQoL-20 and the EORTC-QLQ-C30 were used in both study samples, it was appropriate to combine and compare the data on these common variables. Patient illness characteristics were also examined. This combined dataset was used to analyze cross-cultural similarities and differences in the patterns of HR-QOL factors.

The data collection procedure was the same in both the Netherlands and Japan: during a consultation visit at the hospital, patients who had already undergone at least some form of treatment (surgery, chemotherapy, etc.) were invited to participate in the study by their physician. Other inclusion criteria were cancer site (lung, colorectal and stomach in Japan; lung, colorectal, breast and prostate in The Netherlands) and the absence of any apparent psychiatric disorder (as judged by the consulted physician). After informed consent procedures were completed, patients received a booklet including the Japanese HRQoL-20 as well as the European EORTC-QLQ-C30 plus various other instruments not reported on in this study.

\section{Patients}

All consecutive patients from the participating oncology departments that fulfilled the inclusion criteria were invited to participate into the study, reflecting the incidence rates of the most common cancer sites in Japan and the Netherlands. The Japanese $(n=265)$ and Dutch $(n=176)$ patient groups differed with respect to cancer site, gender (the Dutch group included more females), and Tumor Node Metastasis (TNM) stage (the Japanese group included more TNM stage IV patients) (Table 1).

\section{Instruments}

Two self-report instruments measured HR-QOL: the 20-item Health Related Quality of Life questionnaire (HRQoL-20) [5], and the 30-item European Organization for Research and Treatment of Cancer Core Quality of Life Questionnaire (EORTC-QLQ-C30) [2].

The HRQoL-20 was developed in Japan and measures generic HR-QOL. It comprises 20 items from the physical, psychological, and social domains (see Appendix). Items can be scored by marking one of three available categories (coded 1, 2 or 3 , relating to a negative, intermediate, or positive response to the item, respectively). In addition to the 3 subscales measuring physical ( 8 items), psychological (8 items) and social (4 items) QOL, a measure of general QOL is derived by calculating an overall composite HR-QOL score based on the average of all 20 items [13]. Results for each scale are presented as the mean score of the items of the scale, with higher scores representing better QOL. The HRQoL-20 has been used in research with healthy subjects [11], and with cancer and non-cancer patients [14]. The Dutch adaptation was constructed after a forward and backward translation procedure (from English).

The EORTC-QLQ-C30 is a 30-item questionnaire developed to assess the QOL of cancer patients. It has been translated and validated into 
Table 1. Demographic and illness characteristics of Japanese and Dutch patients

\begin{tabular}{|c|c|c|c|c|c|c|}
\hline & \multicolumn{2}{|c|}{$\begin{array}{l}\text { Japanese patients } \\
\mathrm{N}=265\end{array}$} & \multicolumn{2}{|c|}{$\begin{array}{l}\text { Dutch patients } \\
\mathrm{N}=176\end{array}$} & \multirow[t]{2}{*}{$\chi^{2}[\mathrm{df}]^{\mathrm{a}}$} & \multirow[t]{2}{*}{$p$} \\
\hline & $\mathrm{N}$ & $\%$ & $\mathrm{~N}$ & $\%$ & & \\
\hline Gender & & & & & $\chi^{2}=23.00[1]$ & 0.001 \\
\hline Female & 66 & 24.9 & 83 & 47.1 & & \\
\hline Male & 199 & 75.1 & 93 & 52.9 & & \\
\hline Cancer site & & & & & $\chi^{2}=300.92[1]$ & $<0.001$ \\
\hline Stomach & 82 & 30.9 & - & - & & \\
\hline Lung & 172 & 64.9 & 30 & 17.2 & & \\
\hline Breast & - & - & 61 & 34.7 & & \\
\hline Colorectal & 11 & 4.2 & 30 & 17.2 & & \\
\hline Prostate & - & - & 55 & 31.6 & & \\
\hline TNM $^{b}$ stage $^{c}$ & & & & & $\chi^{2}=45.92[3]$ & 0.001 \\
\hline I & 47 & 22.7 & 35 & 22.9 & & \\
\hline II & 14 & 6.8 & 45 & 29.4 & & \\
\hline III & 43 & 20.8 & 39 & 25.5 & & \\
\hline IV & 103 & 49.8 & 34 & 22.2 & & \\
\hline Age (mean; SD) & 63.4 & 11.5 & 62.4 & 11.3 & & N.S \\
\hline
\end{tabular}

${ }^{a} \chi^{2}$ indicates differences between Japanese and Dutch patients.

b TNM stage: Tumor Node Metastasis stage.

${ }^{\mathrm{c}}$ Due to missing values, $\mathrm{N}=207$ (Japanese) and 153 (Dutch).

many languages, among which are Dutch and Japanese [2, 4]. It comprises two overall scales (one on general health and one on overall QOL), five functional subscales (measuring role, emotional, cognitive and social functioning), and nine symptom subscales (measuring specific symptoms). All 21 items specifying certain symptoms are listed in Table 4. The overall QOL and general health scales are scored using a 7-point scale, and all other subscales are scored using a 4-point Likert scale. The overall QOL scale is comprised of the single EORTC-QLQ-C30 item 30 ("How would you rate your overall quality of life during the past week?"). The complete questionnaire can be obtained at the EORTC website (http://www. eortc.be).

Although both instruments are similar in that they measure certain aspects of HR-QOL, however they differ according to their item content, construction and lay-out, the scoring, the number of items and scales.

\section{Data analyses}

Reliability coefficients (Cronbach's $\alpha$ ) were calculated for the two samples with respect to all
HR-QOL scales. To analyze conceptual similarities, Pearson correlation coefficients were computed between the European and Japanese HR-QOL scales, separately for the Japanese and Dutch patient groups.

Differences in HR-QOL between the Japanese and Dutch patient groups were analyzed by a MANOVA followed by independent ANOVA's $(2 \times 2 \times 4)$, using the various QOL scales as dependent variables, the country of origin, gender and TNM-stage as factors, and age as a covariate. Partial $\eta$ (a partial correlation coefficient) was applied as an effect-size parameter to examine the influence of the factors and covariate, in explaining differences in QOL between the Japanese and Dutch patients (squared $\eta$ can be used as a measure of percentage of the variance explained by the specific factor).

Similarity of the HR-QOL construct between Dutch and Japanese patient groups on the level of individual symptoms was analyzed by following the approach of Fayers and Hand [9]. Each specific EORTC-QLQ-C30 symptom item (a-priori defined as a potential 'causal indicator' of HR-QOL) was ranked according to the Pearson correlation coefficient of each item with the overall measure of 
HR-QOL: EORTC-QLQ-C30 item 30. The same procedure was followed for the composite mean score of the HRQoL-20. This was done separately for the Japanese and Dutch patient groups. These results were compared to those of Fayers and Hand as a means of reference.

Finally, similarities between the different rankings of the different patient groups were examined using Spearman's $\rho$ rank correlations.

\section{Results}

As shown in Table 1 distributions of gender, cancer site and TNM stage were significantly related to country of origin, with relatively more male patients in the Japanese group. Stomach and lung cancer were the dominant types of cancer in the Japanese group, while breast and prostate cancer were the dominant types of cancer in the Dutch group. The Japanese group consisted of more TNM stage IV patients. Mean age was not significantly different between the two groups.

Reliability coefficients were calculated for the overall composite scale and the subscales of the Japanese HRQoL-20, and for the overall QOL scale and the functioning scales of the European
EORTC-QLQ instrument. Table 2 presents the Cronbach's $\alpha$ coefficients showing comparable values, with only a few exceptions. The Cronbach's $\alpha$ coefficients for the overall HRQoL-20 and overall EORTC-QLQ scales were good for both samples. Most $\alpha$ values of the subscales were also satisfactorily high, but there were some exceptions: the $\alpha$ coefficient of the HR-QoL-20 social subscale was low in both samples (range 0.38-0.16). Also, the values of the social and cognitive subscales of the EORTC-QLQ were rather low in both patient groups (range 0.470.63 ), only reaching marginal values of reliability.

Conceptual similarities were analyzed by computing the correlations between the Japanese HRQoL-20 scales and the scales of the Western EORTC-QLQ-C30, separately for the Japanese and Dutch patient groups (see Table 2). The patterns of the various correlation coefficients are remarkably similar between the two groups. The EORTC-QLQ-C30 overall QOL scale correlated highly $(r=0.59 ; p<0.001)$ with the overall HRQoL-20 scale in both groups, indicating substantial conceptual equivalence. Similar values were found when comparing the correlations between the physical and emotional EORTC-QLQC30 subscales with the physical and psychological

Table 2. Cronbach's $\alpha$ coefficients of quality of life scales, and correlations between Japanese and European quality of life scales, in Japanese and Dutch patients

\begin{tabular}{|c|c|c|c|c|c|c|c|c|c|c|}
\hline & \multicolumn{10}{|c|}{ HRQoL-20 scales } \\
\hline & \multicolumn{5}{|c|}{ Japanese patients } & \multicolumn{5}{|c|}{ Dutch patients } \\
\hline & $\alpha$ & Phys. & Psych. & Social & Overall & $\alpha$ & Phys. & Psych. & Social & Overall \\
\hline \multicolumn{11}{|l|}{ HRQoL-20 scales ${ }^{\mathrm{a}}$} \\
\hline Physical & 0.76 & - & & & & 0.74 & - & & & \\
\hline Psychological & 0.72 & $0.59 * * *$ & - & & & 0.70 & $0.64 * * *$ & - & & \\
\hline Social & 0.38 & $0.16^{*}$ & $0.30^{* * *}$ & - & & 0.16 & $0.16^{*}$ & $0.27 * * *$ & - & \\
\hline Overall quality of life & 0.81 & $0.80^{* * *}$ & $0.87^{* * *}$ & $0.52^{* * *}$ & - & 0.82 & $0.84 * * *$ & $0.90 * * *$ & $0.44^{* * *}$ & - \\
\hline \multicolumn{11}{|c|}{ EORTC-QLQ-C30 overall scales ${ }^{b}$} \\
\hline Quality of life & 0.85 & $0.55^{* * *}$ & $0.50^{* * *}$ & $0.14^{*}$ & $0.59^{* * *}$ & 0.88 & $0.63 * * *$ & $0.43^{* * *}$ & $0.21 * *$ & $0.59 * * *$ \\
\hline Physical health & 0.65 & $0.47^{* * *}$ & $0.30^{* * *}$ & $0.22 * *$ & $0.47^{* * *}$ & 0.80 & $0.60 * * *$ & $0.35 * * *$ & 0.05 & $0.52 * * *$ \\
\hline \multicolumn{11}{|c|}{ EORTC-QLQ-C30 functioning scales ${ }^{\mathrm{b}}$} \\
\hline Role & 0.86 & $0.46^{* * *}$ & $0.38^{* * *}$ & 0.10 & $0.46^{* * *}$ & 0.79 & $0.57 * * *$ & $0.29 * * *$ & -0.04 & $0.46^{* * *}$ \\
\hline Emotional & 0.81 & $0.36^{* * *}$ & $0.54 * * *$ & 0.10 & $0.49^{* * *}$ & 0.87 & $0.55 * * *$ & $0.62 * * *$ & $0.19 *$ & $0.64 * * *$ \\
\hline Cognitive & 0.47 & $0.39^{* * *}$ & $0.30^{* * *}$ & $0.22 * *$ & $0.42 * * *$ & 0.61 & $0.43^{* * *}$ & $0.38^{* * *}$ & $0.18^{*}$ & $0.47^{* * *}$ \\
\hline Social & 0.63 & $0.27 * * *$ & $0.30^{* * *}$ & -0.07 & $0.26^{* * *}$ & 0.58 & $0.46^{* * *}$ & $0.39 * * *$ & 0.01 & $0.46^{* * *}$ \\
\hline
\end{tabular}

$* p<0.05 ; * * p<0.01 ; * * * p<0.001$.

a HRQoL-20: Health Related Quality of Life questionnaire-20.

${ }^{\mathrm{b}}$ EORTC-QLQ-C30: European Organization for Research and Treatment of Cancer Core Quality of Life Questionnaire C-30. 
HRQoL-20 scales. An exception to these observed coherent patterns is the correlation between the subscales assessing the social domain of the Japanese and European instruments. As might be expected from the low reliability values of these subscales, there was no substantial correlation between these subscales of the two instruments.

Not shown in Table 2 are the correlations between the physical health and emotional scale of the EORTC-QLQ-C30, but they proved to be low: $r=0.17(p<0.001)$ in the Japanese and $r=0.36$ $(p<0.001)$ in the Dutch patient groups, respectively.

\section{Differences in quality of life scores}

All the scores of the HR-QOL scales (see Table 3) were entered into a single MANOVA analysis using country, gender, and TNM-stage as factors and age as a covariate. Country $(F=3.80 ; d f=19,292$; $p<0.001)$, gender $(F=3.08 ; \mathrm{df}=19,292 ; p<0.001)$ and age $(F=4.03 ; \mathrm{df}=19,292 ; p<0.001)$ were all significantly related to the level of QOL. In addition, the interaction between country, gender, and TNM-stage proved to be significant, although to a much lesser degree $(F=1.48 ; \mathrm{df}=57,882 ; p<0.05)$. Table 3 shows the mean values of all the HR-QOL scales (as used in the MANOVA) for the Japanese and Dutch patient groups, including some details of the results of the univariate ANOVA's on these scores.

As Table 3 shows, the absolute values on the Japanese HRQoL-20 and on the Western EORTC-QLQ-C30 overall and functioning scales were all higher (meaning a better QOL) in the

Table 3. Differences in quality of life scores between Japanese and Dutch patients

\begin{tabular}{|c|c|c|c|c|c|c|c|c|}
\hline & \multicolumn{2}{|c|}{ Japanese patients } & \multicolumn{2}{|c|}{ Dutch patients } & \multicolumn{4}{|c|}{ Effect size (Partial $\eta, F$-test) ${ }^{\mathrm{d}}$} \\
\hline & $\mathrm{M}$ & $\mathrm{SD}$ & M & $\mathrm{SD}$ & Country & Gender & Age & Stage \\
\hline \multicolumn{9}{|l|}{ HRQoL-20 scales a } \\
\hline Physical & 2.19 & 0.4 & 2.31 & 0.4 & $0.16^{* *}$ & - & - & $-0.20 * *$ \\
\hline Psychological & 2.01 & 0.4 & 2.37 & 0.4 & $0.34 * * *$ & - & - & $-0.16^{*}$ \\
\hline Social & 2.40 & 0.4 & 2.60 & 0.4 & $0.16^{* *}$ & - & - & - \\
\hline Overall quality of life & 2.16 & 0.3 & 2.44 & 0.4 & $0.30^{* * *}$ & - & - & $-0.19^{* *}$ \\
\hline \multicolumn{9}{|c|}{ EORTC-QLQ-C30 overall scales ${ }^{b}$} \\
\hline Quality of life & 52.74 & 24.2 & 71.13 & 19.0 & $0.27 * * *$ & - & - & $-0.18^{*}$ \\
\hline Physical health & 73.23 & 25.2 & 78.60 & 20.6 & - & - & $-0.26^{* * *}$ & $-0.17^{*}$ \\
\hline \multicolumn{9}{|c|}{ EORTC-QLQ-C30 functioning scales b } \\
\hline Role & 68.14 & 30.9 & 71.27 & 28.2 & - & - & - & $-0.16^{*}$ \\
\hline Emotional & 70.86 & 21.3 & 78.16 & 22.3 & - & - & - & - \\
\hline Cognitive & 75.06 & 21.5 & 82.24 & 20.3 & - & $0.12 *$ & $-0.12 *$ & - \\
\hline Social & 70.79 & 26.5 & 80.24 & 22.0 & - & - & $-0.16^{* *}$ & - \\
\hline \multicolumn{9}{|c|}{ EORTC-QLQ-C30 symptom scales ${ }^{c}$} \\
\hline Fatigue & 37.51 & 25.4 & 35.00 & 25.0 & - & - & - & - \\
\hline Nausea & 6.23 & 15.9 & 8.04 & 16.8 & - & - & - & - \\
\hline Pain & 23.90 & 27.2 & 20.29 & 23.8 & - & - & - & $-0.18^{*}$ \\
\hline Dyspnea & 26.62 & 29.3 & 17.84 & 29.3 & - & - & - & $-0.16^{*}$ \\
\hline Insomnia & 26.13 & 28.8 & 27.02 & 31.9 & - & - & - & - \\
\hline Appetite loss & 23.68 & 30.0 & 13.53 & 26.8 & $0.14^{*}$ & $0.13^{*}$ & - & $-0.17^{*}$ \\
\hline Constipation & 21.04 & 27.0 & 11.58 & 20.4 & $0.14^{*}$ & - & - & - \\
\hline Diarrhea & 12.37 & 20.9 & 12.18 & 23.8 & - & - & - & - \\
\hline Financial difficulties & 25.00 & 26.8 & 8.63 & 21.4 & $0.23 * * *$ & - & $-0.22 * * *$ & - \\
\hline
\end{tabular}

HRQoL-20: Health Related Quality of Life questionnaire-20; EORTC-QLQ-C30: European Organization for Research and Treatment of Cancer Core Quality of Life Questionnaire C-30.

a Scores can range from 1 to 3 with higher scores indicating better QOL.

b Scores can range from 0 to 100 with higher scores indicating better QOL functioning.

${ }^{\mathrm{c}}$ Scores can range from 0 to 100 with higher scores indicating more severe symptoms.

${ }^{\mathrm{d}}$ Only significant partial correlations are shown $\left({ }^{*} p<0.05, * * p<0.01, * * * p<0.001\right)$. 
Dutch sample. For all the HRQoL-20 scales and for the EORTC-QLQ-C30 overall QOL scale, these differences were significantly related to country. In case of the physical, psychological and overall subscales of the HRQoL-20 and also of the EORTC-QLQ-C30's overall scales and role functioning subscale, these differences were also significantly but negatively related to TNM-stage (if the illness invasion level was worse, then QOL values were lower).

All scores on the EORTC-QLQ-C30 symptom scales were higher (meaning more symptoms) in the Japanese patient group (except for nausea and insomnia). Pain, dyspnea and appetite loss were significantly related to TNM-stage, and appetite loss, constipation and financial difficulties were significantly related to country. A few statistically significant interaction effects were found, but they were rather small: one Country $\times$ TNM-stage interaction for the EORTC-
QLQ-C30 dyspnea scale $(p<0.05)$, and four Country $\times$ Gender $\times$ TNM-stage interactions: for the HRQoL-20 social scale $(p<0.01)$, and the EORTC-QLQ-C30 scales of overall health, emotional well-being and constipation (all $p<0.05$ ).

\section{Causal indicators' of quality of life}

Information on the possible (theoretical) causal relationship between each specific a-priori indicator with overall HR-QOL can be found in Table 4. Pearson correlations of each of the 21 individual EORTC-QLQ-C30 symptom items with the two different measures of overall QOL (EORTC-QLQC30 item 30 and average HRQoL-20) are presented separately for the Japanese and Dutch samples. In addition, the rankings of these symptom items are presented. The rankings of items in the first column were based on their correlations with the overall EORTC-QLQ-C30 scale as was

Table 4. Correlations and rankings (in parentheses) between specific EORTC-QLQ-C30 symptom items and two overall measures of quality of life

\begin{tabular}{|c|c|c|c|c|c|c|}
\hline \multirow[t]{2}{*}{ Ranked EORTC items } & \multicolumn{2}{|c|}{$\begin{array}{l}\text { Correlation with overall } \\
\text { EORTC }^{\mathrm{a}}\end{array}$} & \multicolumn{2}{|c|}{$\begin{array}{l}\text { Correlation with overall } \\
\text { HRQoL- } 20^{\mathrm{b}}\end{array}$} & \multicolumn{2}{|c|}{$\begin{array}{l}\text { Correlations with overall } \\
\text { EORTC }\end{array}$} \\
\hline & Japanese & Dutch & Japanese & Dutch & Fayers and Hand ${ }^{c}$ & Fayers and Hand ${ }^{\mathrm{d}}$ \\
\hline Lack concentration (Q20) & $-0.46(1)$ & $-0.44(3)$ & $-0.38(9)$ & $-0.49(8)$ & $-0.51(7)$ & $-0.54(5)$ \\
\hline Need rest (Q10) & $-0.45(2)$ & $-0.40(6)$ & $-0.51(3)$ & $-0.47(9)$ & $-0.63(3)$ & $-0.56(4)$ \\
\hline Insomnia (Q11) & $-0.43(3)$ & $-0.35(8)$ & $-0.57(1)$ & $-0.41(12)$ & $-0.46(11)$ & $-0.41(10)$ \\
\hline Family life (Q26) & $-0.43(3)$ & $-0.25(14)$ & $-0.29(14)$ & $-0.34(14)$ & $-0.51(7)$ & $-0.53(6)$ \\
\hline Tiredness (Q18) & $-0.40(4)$ & $-0.39(7)$ & $-0.49(4)$ & $-0.55(3)$ & $-0.66(2)$ & $-0.60(3)$ \\
\hline Depressed (Q24) & $-0.40(4)$ & $-0.45(2)$ & $-0.39(8)$ & $-0.62(1)$ & $-0.57(4)$ & $-0.61(2)$ \\
\hline Pain interference (Q19) & $-0.39(5)$ & $-0.34(9)$ & $-0.46(7)$ & $-0.47(9)$ & $-0.49(9)$ & $-0.45(8)$ \\
\hline Appetite (Q13) & $-0.35(6)$ & $-0.42(4)$ & $-0.51(3)$ & $-0.53(5)$ & $-0.56(5)$ & $-0.45(8)$ \\
\hline Worried (Q22) & $-0.35(6)$ & $-0.29(12)$ & $-0.37(10)$ & $-0.50(7)$ & $-0.50(8)$ & $-0.54(5)$ \\
\hline Weakness (Q12) & $-0.34(7)$ & $-0.53(1)$ & $-0.53(2)$ & $-0.59(2)$ & $-0.70(1)$ & $-0.63(1)$ \\
\hline Social activities (Q27) & $-0.33(8)$ & $-0.41(5)$ & $-0.19(16)$ & $-0.44(10)$ & $-0.56(5)$ & $-0.53(6)$ \\
\hline Shortness of breath (Q8) & $-0.32(9)$ & $-0.34(9)$ & $-0.48(5)$ & $-0.35(13)$ & $-0.42(13)$ & $-0.35(12)$ \\
\hline Tense (Q21) & $-0.32(9)$ & $-0.34(9)$ & $-0.33(13)$ & $-0.52(6)$ & $-0.52(6)$ & $-0.56(4)$ \\
\hline Pain (Q9) & $-0.28(10)$ & $-0.33(10)$ & $-0.47(6)$ & $-0.45(10)$ & $-0.47(10)$ & $-0.39(11)$ \\
\hline Memory (Q25) & $-0.28(10)$ & $-0.27(13)$ & $-0.30(14)$ & $-0.31(15)$ & $-0.39(14)$ & $-0.42(9)$ \\
\hline Irritable (Q23) & $-0.26(11)$ & $-0.40(6)$ & $-0.48(5)$ & $-0.54(4)$ & $-0.46(11)$ & $-0.49(7)$ \\
\hline Nausea (Q14) & $-0.25(12)$ & $-0.30(11)$ & $-0.36(11)$ & $-0.34(14)$ & $-0.43(12)$ & $-0.41(10)$ \\
\hline Constipation (Q16) & $-0.24(13)$ & $-0.13(15)$ & $-0.27(15)$ & $-0.29(16)$ & $-0.34(16)$ & $-0.28(14)$ \\
\hline Vomiting (Q15) & $-0.20(14)$ & $-0.11(17)$ & $-0.27(15)$ & $-0.31(15)$ & $-0.35(15)$ & $-0.25(15)$ \\
\hline Financial problems (Q28) & $-0.20(14)$ & $-0.09(18)$ & $-0.35(12)$ & $-0.31(15)$ & $-0.32(17)$ & $-0.31(13)$ \\
\hline Diarrhea (Q17) & $-0.08(15)$ & $-0.21(16)$ & $-0.14(17)$ & $-0.21(17)$ & $-0.28(18)$ & $-0.20(16)$ \\
\hline
\end{tabular}

a Overall QOL scale EORTC as defined by European Organization for Research and Treatment of Cancer Core Quality of Life Questionnaire C-30 item 30

${ }^{\mathrm{b}}$ Overall QOL scale HRQoL-20 as a composite score derived from the Health Related Quality of Life questionnaire-20.

${ }^{\mathrm{c}}$ Data of Norwegian head and neck cancer patients as reported by Fayers Nd Hand (10).

${ }^{\mathrm{d}}$ Data of Danish primary breast cancer patients as reported by Fayers and Hand (10). 
found in the Japanese patients group. Relatively high correlations suggest that an item belongs to the group of indicators that define core aspects of general HR-QOL. Relatively low correlations may point to a 'causal' relationship between the specific item and overall HR-QOL. Although such an item can 'influence' the level of QOL, the reverse is not necessarily true. Taken as a single symptom such items are not suitable to be included in the definition of QOL as a construct.

Fayers and Hand [9] published similar correlations in a study on Norwegian patients with head and neck cancer and Danish women with breast cancer. Therefore, the correlations from their study can be used as a reference and they are also shown in Table 4. In the Japanese patient group, EORTCQLQ-C30 items ('lack of concentration,' 'the need to rest,' 'insomnia,' 'interference with family life,' 'tiredness,' and 'depression') showed relatively high correlations with the overall EORTC-QOL-C30 score. Because of these substantial associations, these items can be conceptualized (at least for the Japanese group) as belonging to the core concept of HR-QOL. The items with low rankings in this sample ('diarrhea,' 'financial problems,' 'vomiting,' 'constipation,' and 'nausea') showed relatively low associations with the overall HR-QOL, and can therefore be conceived as belonging to the category of causal indicators of QOL.
In comparing the six rankings in Table 4 on the Japanese patient group, the Dutch group and the groups of Fayers and Hand, many similarities emerge but also some discrepancies can be observed. Table 5 shows Spearman's rank correlations of all the combinations of the six available rankings. In general, the rank correlations are statistically significant and mostly very high. Correlations obtained with the EORTC-QLQ-C30 ranged from $\rho=0.63$ to $\rho=0.93$, an indication of a rather good cross-cultural structural equivalence for this instrument with regard to the symptom items. Using the average HRQoL-20 score as a measure of overall QOL, some lower rank correlations were found especially in the Japanese patient group $(\rho=0.41-0.64)$ compared with the Dutch group $(\rho=0.56-0.84)$.

\section{Discussion}

Internationally organized clinical trials on the treatment of cancer patients have stimulated the development of cross-culturally valid QOL instruments, such as the EORTC-QLQ-C30. The use of these instruments facilitates the comparison of outcomes on QOL from different countries and increases their accuracy. Although the integration of information on the various indicators of

Table 5. Spearman's $\rho$ correlations between rankings ${ }^{\mathrm{a}}$ of EORTC-QLQ-C30 symptom items in different groups of patients

\begin{tabular}{|c|c|c|c|c|c|c|}
\hline & \multicolumn{2}{|c|}{ EORTC- QLQ-C30 } & \multicolumn{2}{|c|}{ HRQoL-20 } & \multicolumn{2}{|l|}{ EORTC- QLQ-C30 } \\
\hline & Japanese & Dutch & Japanese & Dutch & Fayers and Hand $^{\mathrm{d}}$ & Fayers and Hand ${ }^{\mathrm{e}}$ \\
\hline \multicolumn{7}{|l|}{ EORTC-QLQ-C30 } \\
\hline Japanese patients & - & & & & & \\
\hline Dutch patients & $0.63^{* *}$ & - & & & & \\
\hline \multicolumn{7}{|l|}{ HRQoL-20 ${ }^{c}$} \\
\hline Japanese patients & $0.54 * *$ & $0.63^{* *}$ & - & & & \\
\hline Dutch patients & $0.56^{* *}$ & $0.83^{* * *}$ & $0.64 * *$ & - & & \\
\hline \multicolumn{7}{|l|}{ EORTC-QLQ-C30 b } \\
\hline Fayers and Hand ${ }^{\mathrm{d}}$ & $0.74 * * *$ & $0.80^{* * *}$ & $0.51^{*}$ & $0.84 * * *$ & - & \\
\hline Fayers and Hand ${ }^{\mathrm{e}}$ & $0.70^{* * *}$ & $0.73^{* * *}$ & 0.41 & $0.85^{* * *}$ & $0.92^{* * *}$ & - \\
\hline
\end{tabular}

${ }^{*} p<0.05 ;{ }^{* *} p<0.01 ;{ }^{* * *} p<0.001$;

${ }^{\text {a }}$ See ranked correlations as depicted in Table 4.

${ }^{\mathrm{b}}$ Overall QOL scale EORTC as defined by European Organization for Research and Treatment of Cancer Core Quality of Life Questionnaire C-30 item 30.

${ }^{c}$ Overall QOL scale HRQoL-20 as a composite score derived from the Health Related Quality of Life questionnaire-20.

${ }^{\mathrm{d}}$ Data of Norwegian head and neck cancer patients as reported by Fayers and Hand (10).

${ }^{\mathrm{e}}$ Data of Danish primary breast cancer patients as reported by Fayers and Hand (10). 
intercultural equivalence of QOL instruments is important and valuable, it is often a slow process and seldom the main objective of QOL studies. Comparing the conceptual equivalence of HR-QOL in Japanese and Dutch cancer patients was a major goal of the present study.

Examining the associations of HR-QOL outcomes with variables such as country of origin and illness characteristics was also an important part of the study. Most of the overall, functioning and domain scales showed internal consistency values that were sufficiently high, regardless of the origin of the instrument or country of origin of the patient group. Exceptions to this rule were the $\alpha$ coefficients found for the social and cognitive scales. Values of the overall scales were comparable to results from other studies $[3,15]$.

Although the overall EORTC-QLQ-C30 scale and the overall HRQoL-20 measure general HRQOL quite differently, the cross-correlations of these overall scales showed a substantial and robust cross-cultural equivalence of the QOL concept. We can only partly confirm the observations of Kobayashi et al. [4] of relatively high correlations in Japanese patients between the somatic and emotional scales of the EORTCQLQ-C30 and Aaronson et al.'s [2] relatively lower correlations of these scales in European patients. We found a high correlation between the Physical and Psychological scales of the HRQoL20 , but we did so for both patients groups, irrespective of country of origin. Comparison of our results with research findings from the field of general subjective well-being (SWB) shows some similarities and some differences. As Diener et al. $[16,17]$ reported in a review of measurement validity across cultures, that although cross cultural comparisons of SWB have certainly some degree of validity, some authors report evidence of a slightly different structure for SWB items across cultures. Our results, while using measures of wellbeing in a health-related setting, did only partly support these observations. Particularly measurements in the physical and psychological domains indicated a good degree of conceptual equivalence, but some problems were encountered in the social domain.

Ranking the 21 EORTC QLQ-C30 symptom items according to their correlations with overall measures of HR-QOL has enabled the evaluation of the a-priori classification of symptom items as 'causal indicators'. The various rankings showed substantial similarities, but some discrepancies could also be observed.

A consequence of the procedure of this study was the use of consecutive samples of patients. This limited our possibilities to untangle some of the confounded factors. Some patient characteristics like TNM stage or disease site are related to country of origin. A study using a controlled design is needed to corroborate our main conclusions. In addition, in order to be able to evaluate the impact of these groups of symptoms in cancer clinical trials, studies with a longitudinal design are needed.

Dean [18] pointed out how cultural differences in explanatory models of cancer experience shape the responses of patients to their illness, participation in screening, compliance with treatment, and their relationship with health professionals. The HRQoL-20 developed in Japan, clearly contains items that reflect Japanese explanatory models about patients' responses to cancer. For example, the HRQoL-20 item that asks about whether the respondent has "taken up gardening" or "taking care of a pet" refers to social isolation in the Japanese culture, as it reflects turning away from humans. In North American or European culture, taking care of pets or taking up gardening, as a response to facing cancer would be viewed as a constructive coping response to a taxing situation.

The EORTC QLQ-C30 has been used in a recent study that examined QOL in a multicultural population of cancer patients from Asian and nonAsian Pacific Islanders [19]. The authors report comparable results and conclude that the EORTCQLQ-C30 seems viable for the multi-cultural samples examined. Factor analytic techniques applied to the patients' scores on the EORTC-QLQC30 items supported the dimensions as defined by its developers, especially regarding factors reflecting the concept of social support. In our samples (Dutch and Japanese), we too found support for the viability of the EORTC QLQ-C30, with some reservations about dimensions that purport to assess social functioning. This social domain certainly necessitates further research. Ishikawa et al. [20] and Kaplan et al. [12] studied physician-patient communication patterns in a Japanese cancer setting. They found that these patterns have a 
strong biomedical emphasis compared to Western patient-physician communication patterns [21], illustrating this issue.

In summary, the present study underlines the cross-cultural viability of the EORTC-QLQ-C30 in samples of Dutch and Japanese patients with various types of cancer. The concepts of 'causal indicators' and 'effect indicators', introduced by Fayers and Hand [9], were also found to be relevant in these patient groups. Future research assessing QOL in Japanese and Dutch samples would benefit from using additional questionnaires (e.g., IPQ-R, see http://www.uib.no/ipq) in order to shed light on explanatory models or illness representations and patients' communication style preferences in relation to QOL. This additional information could also be of help in shaping potential interventions on illness perceptions of these patients [22]. Longitudinal designs would be instrumental in clarifying the issue of causal and effect indicators in this type of research.

\section{Acknowledgements}

Part of this study was made possible by financial support of Pfizer Foundation of Japan.We like to thank Dr. H. Vos-Westerman, Dr. R. Bevers, Dr. L. Willems, Dr. A. Dingemans, Dr. R.A.E.M. Tollenaar, Dr. G.G. Kenter and Dr. F.W. Dekker for their support, and Josefine Pouw, Femke Blonk, Elske van den Berg, Emma Tiesma and Wil den Heyer for their assistance in data acquisition.

\begin{tabular}{|c|c|c|}
\hline \multicolumn{3}{|c|}{ Appendix } \\
\hline $\begin{array}{l}\text { Health } \\
\text { (HRQoL }\end{array}$ & $\begin{array}{l}\text { Related } \\
-20)\end{array}$ & Quality of Life questionnaire items \\
\hline 1 & $\mathrm{Ph}$ & $\begin{array}{l}\text { How would you describe your } \\
\text { state of health? }\end{array}$ \\
\hline 2 & $\mathrm{Ph}$ & How often do you feel tired? \\
\hline 3 & Ps & How would you describe your mood? \\
\hline 4 & $\mathrm{Ph}$ & $\begin{array}{l}\text { Are you able to do the physical activities } \\
\text { you would like to do? }\end{array}$ \\
\hline 5 & Ps & Do you have worries in everyday live? \\
\hline 6 & Ps & Can you handle stress? \\
\hline 7 & $\mathrm{Ph}$ & How often do you have swollen legs? \\
\hline 8 & $\mathrm{Ph}$ & How often are you in physical pain? \\
\hline 9 & Ps & $\begin{array}{l}\text { How often do you wonder if your } \\
\text { illness is incurable? }\end{array}$ \\
\hline 10 & Ps & How often do you feel irritated? \\
\hline
\end{tabular}

\begin{tabular}{|c|c|c|}
\hline \multicolumn{3}{|c|}{ Appendix Continued } \\
\hline 11 & Ps & How often do you feel ill? \\
\hline 12 & $\mathrm{Ph}$ & $\begin{array}{l}\text { How often do you feel as if you } \\
\text { were suffocating? }\end{array}$ \\
\hline 13 & So & How often do you feel lonely? \\
\hline 14 & $\mathrm{Ph}$ & Do you have a good appetite? \\
\hline 15 & $\mathrm{Ph}$ & Do you sleep well? \\
\hline 16 & So & $\begin{array}{l}\text { Do you feel that members of your family } \\
\text { or other people need you? }\end{array}$ \\
\hline 17 & Ps & $\begin{array}{l}\text { How often are you worried about } \\
\text { the cost of living? }\end{array}$ \\
\hline 18 & So & $\begin{array}{l}\text { How would you describe your } \\
\text { relationship with your family, } \\
\text { friends, and neighbors? }\end{array}$ \\
\hline 19 & So & Do you have pets or do you like gardening? \\
\hline 20 & Ps & Are you satisfied with your current life? \\
\hline
\end{tabular}

$\mathrm{Ph}$, Ps or So refer to items belonging to the Physical, Psychological and Social subscales.

\section{References}

1. Naito M, Nakayama T, Fukuhara S. Quality of life assessment and reporting in randomized controlled trials: A study of literature published from Japan. Health Qual Life Outcomes 2004; 2: 31-37.

2. Aaronson NK, Ahmedzai S, Bergman B, et al., for the EORTC Study Group on QOL. The European Organization for Research and Treatment of Cancer QLQ-C30: A quality-of-life instrument for use in international clinical trials in oncology. J Natl Cancer Inst 1993; 85: 365-376.

3. Kobayashi K, Green J, Shimonagayoshi M, et al. Validation of the care notebook for measuring physical, mental and life well-being of patients with cancer. Qual Life Res 2005; 14: 1035-1043.

4. Kobayashi K, Takeda F, Teramukai S, et al. A crossvalidation of the European Organization for Research and Treatment of Cancer QLQ-C30 (EORTC QLQ-C30) for Japanese with lung cancer. Eur J Cancer 1998; 34: 810815.

5. Yamaoka K, Ogoshi K, Haruyama K, et al. Validity of the Japanese version of the questionnaire for quality of life measurement (QOL20). Int Med J 1998; 5: 23-29.

6. Yamaoka K, Hayashi F, Hayashi C, et al. A Japanese version of the questionnaire for quality of life measurement. Ann Cancer Res Ther 1994; 3: 45-53.

7. Aaronson NK. Assessing the quality of life of patients with cancer: East meets west. Eur J Cancer 1998; 34: 767-769.

8. Hui C, Triandis HC. Measurement in cross-cultural psychology: A review and comparison of strategies. Cross Cult Psychol 1985; 16: 131-152.

9. Fayers PM, Hand DJ. Factor analysis, causal indicators and quality of life. Qual Life Res 1997; 6: 139-150.

10. Fayers PM, Hand DJ, Bjordal K, Groenvold M. Causal indicators in quality of life research. Qual Life Res 1997; 6: 393-406. 
11. Yamaoka K, Hayashi F, Hayashi C, Shigehisa T, Watanabe M. Influence of personality on quality of life measurement. Jap J Health Psychol 1996; 9: 11-20.

12. Kaplan RM, Shigehisa T, Oldenburg B. Health policy, international variation and doctor-patient interaction. Jap J Health Psychol 2003; 4: 23-49.

13. Shigehisa T. Eysenckian personality traits and healthrelated quality of life in patients with stomach, colorectal or breast cancer. Jap J Health Psychol 2003; 10: 19-32.

14. Yamaoka K, Shigehisa T, Ogoshi K, et al. Health-related quality of life varies with personality types: A comparison among cancer patients, non-cancer patients and healthy individuals in a Japanese population. Qual Life Res 1998; 7 : 535-544.

15. Yamaoka K, Takeda F, Shigehisa T, et al. Health-related quality of life in Japanese lung cancer patients as determined by two questionnaires: The HRQOL-20 and the EORTC QLQ-C30. Ann Cancer Res Ther 2003; 11: 31-46.

16. Diener E, Oishi S, Lucas R. Personality, culture, and Subjective well-being: Emotional and cognitive evaluations of life. Annu Rev Psychol 2003; 54: 403-425.

17. Diener E, Suh E, Smit H, Shao L. National differences in subjective well-being: Why do they occur?. Soc Indic Res 1995; 34: 7-32.
18. Dean S. Explanatory models and attitudes towards cancer in different cultures. Lancet Oncol 2004; 5: 119-124.

19. Gotay CC, Blaine D, Haynes SN, Holup J, Pagano IS. Assessment of quality of life in a multicultural cancer patient population. Psychol Assessm 2002; 14: 439-450.

20. Ishikawa H, Takayama T, Yamazaki Y, Seki Y, Katsumata N. Physician-patient communication and patient satisfaction in Japanese cancer consultations. Soc Sci Med 2002; 55: 301-311.

21. Kaptein AA, Kleijn WC, Nortier H. Doctor-patient communication: A European perspective. Ann Cancer Res Ther 2003; 11: 7-14.

22. Petrie KJ, Cameron LD, Ellis CJ, Buick D, Weinman J. Changing illness perceptions after myocardial infarction: An early intervention trial. Psychosom Med 2002; 64: 580586.

Address for correspondence: Wim Chr. Kleijn, Leiden University Medical Center, Medical Psychology, P.O. Box 9555, 2300 RB Leiden, The Netherlands

Phone: +31-71-527-5242; Fax: +31-71-527-3668

E-mail: w.c.kleijn@lumc.nl 